\title{
Reduced sensitivity of the $(d, p)$ cross sections to the deuteron model beyond adiabatic approximation
}

\author{
M. Gómez-Ramos ${ }^{1}$ and N.K. Timofeyuk ${ }^{2}$ \\ ${ }^{1}$ Departamento de FAMN, Universidad de Sevilla, Apartado 1065, 41080 Sevilla, Spain \\ ${ }^{2}$ Department of Physics, Faculty of Engineering and Physical Sciences, \\ University of Surrey Guildford, Surrey GU2 7XH, United Kingdom
}

(Dated: June 26, 2018)

\begin{abstract}
It has recently been reported [Phys. Rev. Lett. 117, 162502 (2016)] that $(d, p)$ cross sections can be very sensitive to the $n-p$ interactions used in the adiabatic treatment of deuteron breakup with nonlocal nucleon-target optical potentials. To understand to what extent this sensitivity could originate in the inaccuracy of the adiabatic approximation we have developed a leading-order localequivalent continuum-discretized coupled-channel model that accounts for non-adiabatic effects in the presence of nonlocality of nucleon optical potentials. We have applied our model to the astrophysically relevant reaction ${ }^{26 m} \mathrm{Al}(d, p){ }^{27} \mathrm{Al}$ using two different $n$ - $p$ potentials associated with the lowest and the highest $n-p$ kinetic energy in the short-range region of their interaction, respectively. Our calculations reveal a significant reduction of the sensitivity to the high $n-p$ momenta thus confirming that it is mostly associated with theoretical uncertainties of the adiabatic approximation itself. The non-adiabatic effects in the presence of nonlocality were found to be stronger than those in the case of the local optical potentials. These results argue for extending the analysis of the $(d, p)$ reactions, measured for spectroscopic studies, beyond the adiabatic approximation.
\end{abstract}

Introduction. One nucleon transfer in $(d, p)$ reactions is an important source of information about the singleparticle strength in atomic nuclei, quantified by spectroscopic factors and asymptotic normalization coefficients. They are obtained from a comparison of experimental and theoretical cross sections calculated using direct transfer reaction theory and, therefore, are influenced by its uncertainties. The uncertaintities arising due to the input optical potentials and the shape of the mean field that binds the transferred neutron has been known for a very long time. Recently, new theoretical uncertainties have been identified in Ref. [1], associated with the $n-p$ interaction used in adiabatic treatement of deuteron breakup with nonlocal nucleon optical potentials. This work studied the ${ }^{26} \mathrm{Al}(d, p)^{27} \mathrm{Al}$ reaction, measured in [2] to pin down the ${ }^{26} \mathrm{Al}$ destruction by the $(p, \gamma)$ reactions in novae explosions, and used several deuteron models: Hulthén model [3], AV18 [4], Reid soft core [5], CD-Bonn $[6]$ and the chiral effective field theory at N4LO with five different regulators [7]. All these models produce exactly the same deuteron wave functions $\phi_{d}$ and the vertex functions $V_{n p} \phi_{d}$, where $V_{n p}$ is the $n-p$ potential, at the $n-p$ separations $r$ larger than than $2 \mathrm{fm}$. However, the model predictions for these quantities at $0<r<2 \mathrm{fm}$ are very different. This sensitivity to the short-range $n$ - $p$ wave functions (and the corresponding sensitivity to the high $n-p$ momenta) seems puzzling given the relatively low deuteron incoming energies, about $10 \mathrm{MeV}$, for which the $(d, p)$ calculations have been done in [1]. Such sensitivity may indicate that other important effects, associated with $(d, p)$ reaction mechanisms, are missing in these calculations.

In this paper, we show that most of the sensitivity of the $A(d, p) B$ cross sections to the high $n-p$ momenta goes away when deuteron breakup is treated beyond the adiabatic distorted-wave approximation (ADWA). The latter is based on the dominant term in the Weinberg state expansion of the $A+n+p$ wave function, calculated neglecting the couplings to all the other Weinberg components [8]. In ADWA with local $n-A$ and $p$ - $A$ optical potentials, the adiabatic potential $U_{d A}(R)$, given by the sum $U_{n A}(R)+U_{p A}(R)[9]$, does not depend on deuteron model. However, the nonlocal adiabatic potential explicitly depends on the average $n$ - $p$ kinetic energy over the (short) range of their interaction, given by the matrix element $\left\langle T_{n p}\right\rangle_{V} \equiv\left\langle\phi_{d}\left|V_{n p} T_{n p}\right| \phi_{d}\right\rangle /\left\langle\phi_{d}\left|V_{n p}\right| \phi_{d}\right\rangle[1,10-12]$. This matrix element is very sensitive to high $n-p$ momenta, which is reflected in the ADWA cross sections.

We choose the continuum-discretized coupled-channel (CDCC) approach $[13,14]$ to treat deuteron breakup in $A(d, p) B$ reactions beyond the adiabatic aproximation. The CDCC, developed and used for local nucleontarget optical potentials only, in some cases predicts significantly different cross sections than the ADWA does [15-17]. Extending the CDCC to the case of nonlocal $n-A$ and $p$ - $A$ potentials, in principle, could be done on the basis of the exact nonlocal ADWA formalism of Ref. [12]. However, it would involve time-consuming calculations of nonlocal kernels when the $d$-wave component in deuteron is included, making the whole task difficult. For this reason, based on ideas of $[10,11]$ we have developed a leading-order local-equivalent CDCC approximation to have a quick accessment of the role of the high $n$ - $p$ momenta in $(d, p)$ reactions. In the ADWA, the leading order solution deviates from the exact one by about $10 \%$ but the sensitivity to the deuteron model is present in both of them in the same proportions [12], which justifies using of the leading order local-equivalent CDCC for our purposes.

Nonlocal CDCC model. In the CDCC, the wave function $\Phi(\boldsymbol{R}, \boldsymbol{r})$ of the $A+n+p$ system includes expansion over the $n-p$ continuum bins $\phi_{i}(\boldsymbol{r})$. To begin with, we 
assume that the bins represent only the $s$-wave motion and that all spins are neglected. In this case,

$$
\Phi(\boldsymbol{R}, \boldsymbol{r})=\sum_{i=0} \chi_{i}(\boldsymbol{R}) \phi_{i}(\boldsymbol{r})
$$

and channel function $\chi_{i}$ are found from the three-body nonlocal Schrödinger equation given by Eq. (9) of Ref. [11]. In Eq. (1) and everywhere below we assume that $\phi_{0}$ is the deuteron bound state wave function $\phi_{d}$. We assume that nonlocal potentials $\mathcal{U}_{n A}$ and $\mathcal{U}_{p A}$ have the Perey-Buck form [18],

$$
\mathcal{U}_{N A}\left(\boldsymbol{r}, \boldsymbol{r}^{\prime}\right)=H\left(\boldsymbol{r}-\boldsymbol{r}^{\prime}\right) U_{N A}\left[\left(\boldsymbol{r}+\boldsymbol{r}^{\prime}\right) / 2\right],
$$

with the nonlocality factor $H$ of the (small) range $\beta$,

$$
H(x)=\pi^{-3 / 2} \beta^{-3} e^{-(x / \beta)^{2}}
$$

and the formfactor $U_{N A}$ is given by usual Woods-Saxon form. Following the reasoning of [11] it is easy to show that $\chi_{i}$ can be found from the nonlocal coupled equations

$$
\begin{aligned}
& \left(T_{R}+U_{C}(R)-E_{d}\right) \chi_{i}(\boldsymbol{R})= \\
& \quad-\sum_{i^{\prime}} \int d \boldsymbol{s} H(s) \mathcal{V}_{i i^{\prime}}(\boldsymbol{s}, \boldsymbol{R}) \chi_{i^{\prime}}\left(\frac{\alpha_{2} s}{2}+\boldsymbol{R}\right),
\end{aligned}
$$

where $T_{R}$ is the kinetic energy operator, $U_{C}$ is the Coulomb potential energy and $E_{d}$ is the center-of-mass beam energy of the $d-A$ system,

$\mathcal{V}_{i i^{\prime}}(\boldsymbol{s}, \boldsymbol{R})=\sum_{N} \int d \boldsymbol{x} \phi_{i}^{*}\left(\boldsymbol{x}+\alpha_{1} \boldsymbol{s}\right) U_{N A}\left(\frac{\boldsymbol{x}}{2}-\boldsymbol{R}\right) \phi_{i^{\prime}}(\boldsymbol{x})$,

$N$ is $n$ or $p, \alpha_{1}=A /(A+1)$ and $\alpha_{2}=(A+2) /(A+1)$. Because of the short range of $H(s)$ the wave function $\chi_{i^{\prime}}\left(\frac{\alpha_{2} \boldsymbol{s}}{2}+\boldsymbol{R}\right)$ can be represented by the leading-order expansion that retains only spherical components in $s$ [11],

$$
\chi_{i^{\prime}}\left(\frac{\alpha_{2} s}{2}+\boldsymbol{R}\right) \approx \sum_{n=0}^{n_{\max }} \frac{s^{2 n}}{\beta^{2 n}} \gamma_{n} T_{R}^{n} \chi_{i^{\prime}}(\boldsymbol{R})
$$

in which

$$
\gamma_{n}=\frac{(-)^{n}}{n !(2 n+1) ! !}\left(\frac{\mu_{d} \alpha_{2}^{2} \beta^{2}}{4 \hbar^{2}}\right)^{n},
$$

where $\mu_{d}$ is the reduced mass of $A+d$. Then Eqs. (4) become

$$
\begin{aligned}
\left(T_{R}+U_{C}(R)\right. & \left.-E_{d}\right) \chi_{i}(\boldsymbol{R})= \\
& -\sum_{n=0}^{n_{\max }} \gamma_{n} \sum_{i^{\prime}} U_{i i^{\prime}}^{(n)}(\boldsymbol{R}) T_{R}^{n} \chi_{i^{\prime}}(\boldsymbol{R}),
\end{aligned}
$$

with the coupling potentials

$$
U_{i i^{\prime}}^{(n)}(\boldsymbol{R})=\int d \boldsymbol{x}\left[\bar{\phi}_{i}^{(n)}(\boldsymbol{x})\right]^{*}\left[\sum_{N} U_{N A}\left(\frac{\boldsymbol{x}}{2}-\boldsymbol{R}\right)\right] \phi_{i^{\prime}}(\boldsymbol{x})
$$

that contain the modified-by-nonlocality functions

$$
\bar{\phi}_{i}^{(n)}(\boldsymbol{x})=\int d \boldsymbol{s} H(s)\left(\frac{s}{\beta}\right)^{2 n} \phi_{i}\left(\boldsymbol{x}+\alpha_{1} s\right) .
$$

To solve the coupled equations (8) we use the local energy-approximation. In the case of a single channel, this approximation means $T_{R} \approx E-U_{C}-U_{l o c}(R)$ with $U_{l o c}$ obtained from a transcendental equation [18]. For the multichannel CDCC case we introduce a generalization of the local-energy approximation,

$$
T_{R} \chi_{i}(\boldsymbol{R})=\sum_{k}\left[\left(E-U_{C}(R)\right) \delta_{i k}-U_{i k}^{\mathrm{loc}}(\boldsymbol{R})\right] \chi_{k}(\boldsymbol{R}) .
$$

We apply it $n_{\max }$ times to the r.h.s. of Eq. (8) neglecting commutators between $T_{R}$ and $U_{i i^{\prime}}^{\text {loc }}$. For one-channel case, the corrections beyond this assumption, determined by $\beta^{4}$, are very small [11]. Imposing the requirement that the local-equivalent coupling potentials $U_{i i^{\prime}}^{\text {loc }}$ satisfy

$$
\left(T_{R}+U_{C}(R)-E_{d}\right) \chi_{i}(\boldsymbol{R})=-\sum_{i^{\prime}} U_{i i^{\prime}}^{\mathrm{loc}}(\boldsymbol{R}) \chi_{i^{\prime}}(\boldsymbol{R})
$$

we obtain a system of the transcendental matrix equations

$$
\begin{aligned}
f_{i i^{\prime}}^{(0)}-\left(E_{j}-U_{C}\right) \delta_{i j} & +\sum_{k}\left(f_{i i^{\prime}}^{(1)}+\delta_{i k}\right) X_{k i^{\prime}} \\
& +\sum_{k l} f_{i i^{\prime}}^{(2)} X_{k l} X_{l i^{\prime}}+\ldots=0,
\end{aligned}
$$

for

$$
X_{i i^{\prime}}=\left(E_{i^{\prime}}-U_{C}\right) \delta_{i i^{\prime}}-U_{i i^{\prime}}^{\mathrm{loc}}
$$

in which $f_{i j}^{(n)}=\gamma_{n} U_{i i^{\prime}}^{(n)}$. We solve equations (13) us-

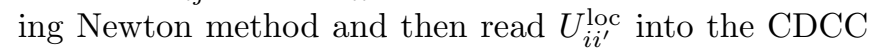
reaction code, which in our case was FRESCO [19].

The scheme described above remains unchanged when all spins are included. We will assume in the following that the target has spin 0, although it can be proved that a non-zero target spin simply introduces an overall factor in the coupling potentials. In the coupling scheme, consistent with FRESCO $\left(\boldsymbol{l}+\boldsymbol{s}_{\boldsymbol{n}}=\boldsymbol{j}_{\boldsymbol{n}}, \boldsymbol{j}_{\boldsymbol{n}}+\boldsymbol{s}_{\boldsymbol{p}}=\boldsymbol{I}\right.$ and $\boldsymbol{L}+\boldsymbol{I}=\boldsymbol{J})$, the bin functions $\phi_{\alpha}$ are labeled by a set of quantum numbers $\alpha=\left\{i, l, j_{n}\right\}$, where $i$ includes both the bin energy and its total angular momentum $I, l$ is the $n-p$ orbital momentum and $j_{n}$ is the total momentum of neutron. The channel functions $\chi_{i L J}$ depend on the $d-A$ relative orbital momentum $L$ and total momentum $J$. We require that the local-equivalent coupling potentials $U_{i i^{\prime} \lambda}^{\text {loc }}$ satisfy

$$
\begin{aligned}
& \left(T_{R}+U_{C}(R)-E_{d}\right) \chi_{i L J}(R)= \\
& \quad-\sum_{i^{\prime} L^{\prime} \lambda} \mathcal{C}_{I^{\prime} L^{\prime} \lambda}^{L I J} U_{i i^{\prime} \lambda}^{\text {loc }}(R) \chi_{i^{\prime} L^{\prime} J}(R),
\end{aligned}
$$

where the (un)primed quantities correspond to the (initial) final state,

$$
\mathcal{C}_{I^{\prime} L^{\prime} \lambda}^{L I J}=(-)^{I+L+J}\left\{\begin{array}{ccc}
L & I & J \\
I^{\prime} & L^{\prime} & \lambda
\end{array}\right\} \hat{L}^{\prime}\left\langle L^{\prime} 0 \lambda 0 \mid L 0\right\rangle
$$


and $\hat{a}=\sqrt{2 a+1}$. The $U_{i i^{\prime} \lambda}^{\text {loc }}$ are also found from a system of transcendental matrix equations

$$
\begin{aligned}
g_{i i^{\prime} \lambda}^{(0)}-\left(E_{i^{\prime}}-U_{C}\right) \hat{I} \delta_{i i^{\prime}} \delta_{\lambda 0}+\sum_{k_{1} l_{1} l_{2} j_{1}}\left(g_{i k_{1} l_{1}}^{(1)}+\hat{I} \delta_{i k_{1}} \delta_{l_{1} 0}\right) \frac{\hat{\lambda}}{\hat{l}_{2}} \mathcal{C}_{j_{1} l_{2} l_{1}}^{\lambda I I_{1}^{\prime}} X_{k_{1} i^{\prime}}^{\left(l_{2}\right)}+\sum_{\substack{k_{1} k_{2} j_{1} j_{2} \\
l_{1} l_{2} l_{3} \Lambda_{1}}} g_{i k_{1} l_{1}}^{(2)} \frac{\hat{\Lambda_{1}}}{\hat{l}_{2}} \mathcal{C}_{j_{1} l_{2} l_{1}}^{\Lambda_{1} I j_{2}} \mathcal{D}_{j_{2} l_{3} \Lambda_{1}}^{\lambda I I^{\prime}} X_{k_{1} k_{2}}^{\left(l_{2}\right)} X_{k_{2} i^{\prime}}^{\left(l_{3}\right)} \\
+\sum_{\substack{k_{1} k_{2} k_{3} j_{1} j_{2} j_{3} \\
l_{1} l_{2} l_{3} l_{4} \Lambda_{1} \Lambda_{2}}} g_{i k_{1} l_{1}}^{(3)} \frac{\hat{\Lambda}_{1}}{\hat{l}_{2}} \mathcal{C}_{j_{1} l_{2} l_{1}}^{\Lambda_{1} I j_{2}} \mathcal{D}_{j_{2} l_{3} \Lambda_{1}}^{\Lambda_{2} I_{3}} \mathcal{D}_{j_{3} l_{4} \Lambda_{2}}^{\lambda I I^{\prime}} X_{k_{1} k_{2}}^{\left(l_{2}\right)} X_{k_{2} k_{3}}^{\left(l_{3}\right)} X_{k_{3} i^{\prime}}^{\left(l_{4}\right)}+\ldots=0
\end{aligned}
$$

with $\mathcal{D}_{I^{\prime} L^{\prime} \lambda}^{L I J}=(-)^{I^{\prime}-I} \hat{L} \hat{L}^{\prime-1} \mathcal{C}_{I^{\prime} L^{\prime} \lambda}^{L I J}$ and $j_{i}$ being the spin of state $k_{i}$, written for

$$
X_{i i^{\prime}}^{(\lambda)}=\left(E_{i^{\prime}}-U_{C}\right) \hat{I}^{\prime} \delta_{i i^{\prime}} \delta_{\lambda 0}-U_{i i^{\prime} \lambda}^{\text {loc }} .
$$

Eqs. (17) now include all necessary angular momentum couplings. They contain functions

$$
\begin{aligned}
g_{i i^{\prime} \lambda}^{(n)}(R) & =\gamma_{n} \sum_{l l^{\prime} j_{n} j_{n}^{\prime}}(-)^{l+s_{n}+s_{p}+j_{n}+j_{n}^{\prime}+I^{\prime}} \hat{I} \hat{I}^{\prime} \hat{j}_{n} \hat{j}_{n}^{\prime} \frac{\hat{\lambda}^{2} \hat{l}}{4 \pi} \\
& \times\left\{\begin{array}{ccc}
j_{n} & j_{n}^{\prime} & \lambda \\
I^{\prime} & I & s_{p}
\end{array}\right\}\left\{\begin{array}{ccc}
j_{n} & j_{n}^{\prime} & \lambda \\
l^{\prime} & l & s_{n}
\end{array}\right\} U_{\alpha \alpha^{\prime} \lambda}^{(n)}(R),
\end{aligned}
$$

determined by the multipoles of the coupling potentials folded between the original $\phi_{i}$ and modified $\bar{\phi}_{i}$ functions:

$$
\begin{aligned}
& U_{\alpha \alpha^{\prime} \lambda}^{(n)}(R)=\int_{0}^{\infty} d x x^{2}\left[\bar{\phi}_{\alpha}^{(n)}(x)\right]^{*}\left[\sum_{N} U_{N A}^{(\lambda)}(x, R)\right] \phi_{\alpha^{\prime}}(x) \\
& U_{N A}^{(\lambda)}(x, R)=2 \pi \int_{-1}^{1} d u U_{N A}\left(\frac{\boldsymbol{x}}{2}-\boldsymbol{R}\right) P_{\lambda}(u),
\end{aligned}
$$

with $u$ being the cosine between $\boldsymbol{x}$ and $\boldsymbol{R}$ and $P_{\lambda}(u)$ the Legendre polynomial.

Application to the ${ }^{26 m} \mathrm{Al}(d, p)^{27} \mathrm{Al}$ reaction. We apply the newly developed local-equivalent $\mathrm{CDCC}$ model to the $(d, p)$ reaction recently measured in inverse kinematics with isomeric ${ }^{26 m} \mathrm{Al}$ beam [20]. Because of the $0^{+}$spin of this isomer transfers to the final ${ }^{27} \mathrm{Al}$ states will involve only one orbital momentum, thus facilitating extraction of spectroscopic factors.

We have performed the CDCC calculations for three incident deuteron energies, 9.2, 25 and $50 \mathrm{MeV}$, typical for the TRIUMF, GANIL and RIKEN facilities. We used the Gianinni-Ricco systematics of energy-independent nonlocal nucleon optical potentials for $N=Z$ targets [21] and two nucleon-nucleon (NN) potentials: Hulthén and RSC. In Ref. [1] the calculations with these potentials gave the lowest and the highest ${ }^{26 g} \mathrm{Al}(d, p)^{27} \mathrm{Al}$ cross sections, respectively. Both $s$ - and $d$-wave continuum bins were used in the calculations. For the reaction at 9.2 $\mathrm{MeV}$ three bins were taken for each component considered equispaced for proton-neutron energies from 0 to 6
$\mathrm{MeV}$ (closed channels start at $6.3 \mathrm{MeV}$ ). At $25 \mathrm{MeV}$, five bins were taken from 0 to $20 \mathrm{MeV}$ (closed channels at $20.97 \mathrm{MeV}$ ) and at $50 \mathrm{MeV}$, four bins from 0 to 44 (closed channels at $44.18 \mathrm{MeV}$ ). Convergence with bin mesh was checked in calculations with local potentials at all energies and with nonlocal potentials at $9.2 \mathrm{MeV}$. We were also made aware that contributions from the closed channels at low $E_{d}$ are negligible [22].

We have calculated the local-equivalent coupling potentials $U_{i j \lambda}^{\text {loc }}(R)$ at each point $R$ from 0 to $50 \mathrm{fm}$ by solving Eq. (17) using the Newton method. The choice of $n_{\max }=3$ was sufficient for $U_{i j \lambda}^{\text {loc }}$ to converge, similar to findings in the one-channel study [11]. For some $d$-wave channels, $n_{\max }=2$ was sufficient. The $U_{i j \lambda}^{\mathrm{loc}}$ have been read into FRESCO which calculated the channel functions $\chi_{i}$ and then the finite range transfer cross sections using the same NN potentials in the transfer vertex. In the case of the RSC, both the $s$ - and $d$-wave deuteron
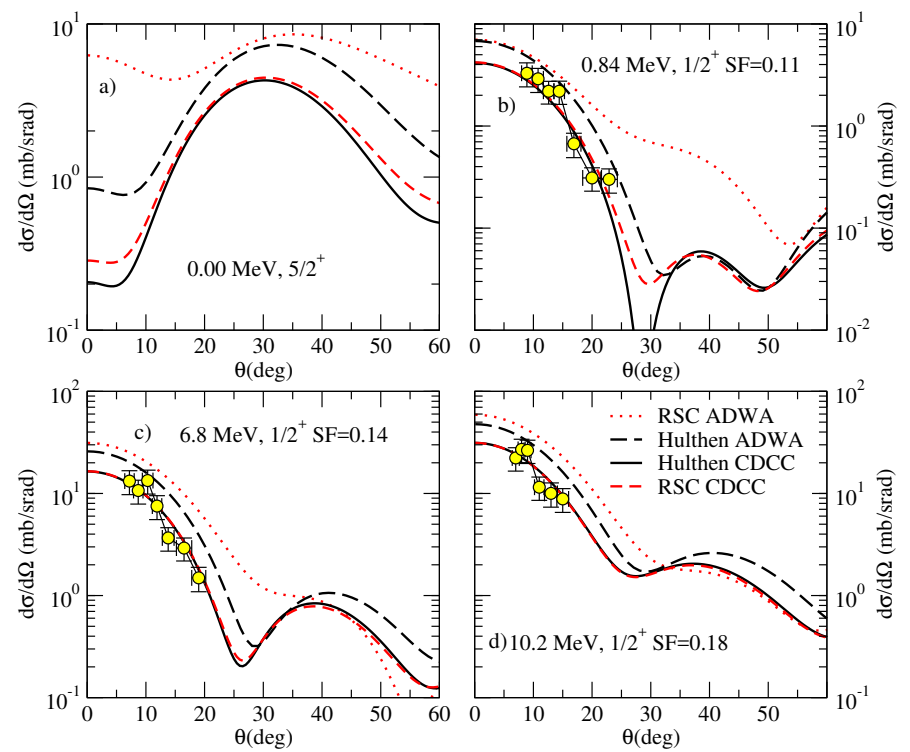

FIG. 1. The differential cross sections of ${ }^{26 m} \mathrm{Al}(d, p){ }^{27} \mathrm{Al}$ at $E_{d}^{\text {lab }}=9.2 \mathrm{MeV}$ for population of the ${ }^{27} \mathrm{Al}\left(5 / 2^{+}\right)$ground state (a) and the excited $1 / 2^{+}$states at $E_{x}=0.84,6.8$ and 10.2 $\mathrm{MeV}(b, c, d)$, respectively. In the cases with experimental data [20] all calculations have been multiplied by the spectroscopic factor obtained for the RSC-CDCC calculation. 
TABLE I. Various ratios of the ${ }^{26 m} \mathrm{Al}(d, p){ }^{27} \mathrm{Al}$ cross sections calculated in the ADWA and CDCC with two different NN potentials, RSC and Hulthén, for $E_{d}^{\mathrm{lab}}=9.2,25$ and $50 \mathrm{MeV}$ and for four final states in ${ }^{27} \mathrm{Al}$. The ratios were calculated at the maxima of cross secitons. All energies are given in $\mathrm{MeV}$.

\begin{tabular}{|c|c|c|c|c|c|c|c|c|c|c|c|c|}
\hline \multirow[b]{2}{*}{$E_{x} \backslash E_{d}^{\mathrm{lab}}$} & \multicolumn{3}{|c|}{$\sigma_{\mathrm{RSC}}^{\mathrm{ADWA}} / \sigma_{\mathrm{H}}^{\mathrm{ADWA}}$} & \multicolumn{3}{|c|}{$\sigma_{\mathrm{RSC}}^{\mathrm{CDCC}} / \sigma_{\mathrm{H}}^{\mathrm{CDCC}}$} & \multicolumn{3}{|c|}{$\sigma_{\mathrm{H}}^{\mathrm{ADWA}} / \sigma_{\mathrm{H}}^{\mathrm{CDCC}}$} & \multicolumn{3}{|c|}{$\sigma_{\mathrm{RSC}}^{\mathrm{ADWA}} / \sigma_{\mathrm{RSC}}^{\mathrm{CDCC}}$} \\
\hline & 9.2 & 25 & 50 & 9.2 & 25 & 50 & 9.2 & 25 & 50 & 9.2 & 25 & 50 \\
\hline 0.00 & 1.17 & 1.38 & 1.76 & 1.04 & 1.08 & 1.05 & 1.71 & 1.80 & 1.55 & 1.81 & 2.35 & 2.60 \\
\hline 0.84 & 1.03 & 1.12 & 2.24 & 1.01 & 0.97 & 1.14 & 1.64 & 1.29 & 1.78 & 1.67 & 1.50 & 3.54 \\
\hline 6.8 & 1.21 & 0.86 & 2.09 & 1.00 & 0.96 & 1.07 & 1.57 & 1.28 & 1.37 & 1.89 & 1.14 & 2.68 \\
\hline 10.2 & 1.24 & 0.81 & 1.69 & 1.00 & 0.96 & 1.05 & 1.52 & 1.27 & 1.10 & 1.89 & 1.08 & 1.76 \\
\hline
\end{tabular}

vertex functions were used. The $\left\langle{ }^{26 m} \mathrm{Al} \mid{ }^{27} \mathrm{Al}\right\rangle$ overlap function was represented by the neutron single-particle wave function, calculated for the Woods-Saxon potential well with the standard radius $r_{0}=1.25 \mathrm{fm}$ and diffusseness $a=0.65 \mathrm{fm}$.

The leading order nonlocal CDCC and ADWA calculations are shown in Fig. 1 and 2 for deuteron incident energies of 9.2 and $50 \mathrm{MeV}$, respectively, and for four final ${ }^{27} \mathrm{Al}$ states: the ground $J^{\pi}=5 / 2^{+}$state and three astrophysically revelant excited $J^{\pi}=1 / 2^{+}$states. In all cases, the CDCC cross sections are significantly lower than the ADWA ones. Their ratio in the maximum, shown in Table I, in most cases is higher than an average value of 1.25 reported for local optical potentials in [17]. The ratio seems to correlate with the neutron separation energy in the final state: for $l=0$ transfer to the final $1 / 2^{+}$state it decreases with excitation energy.

The ADWA cross sections, obtained with Hulthén and RSC, differ up to a factor of two in the maximum (see Table I), which is related to the small and large values of the
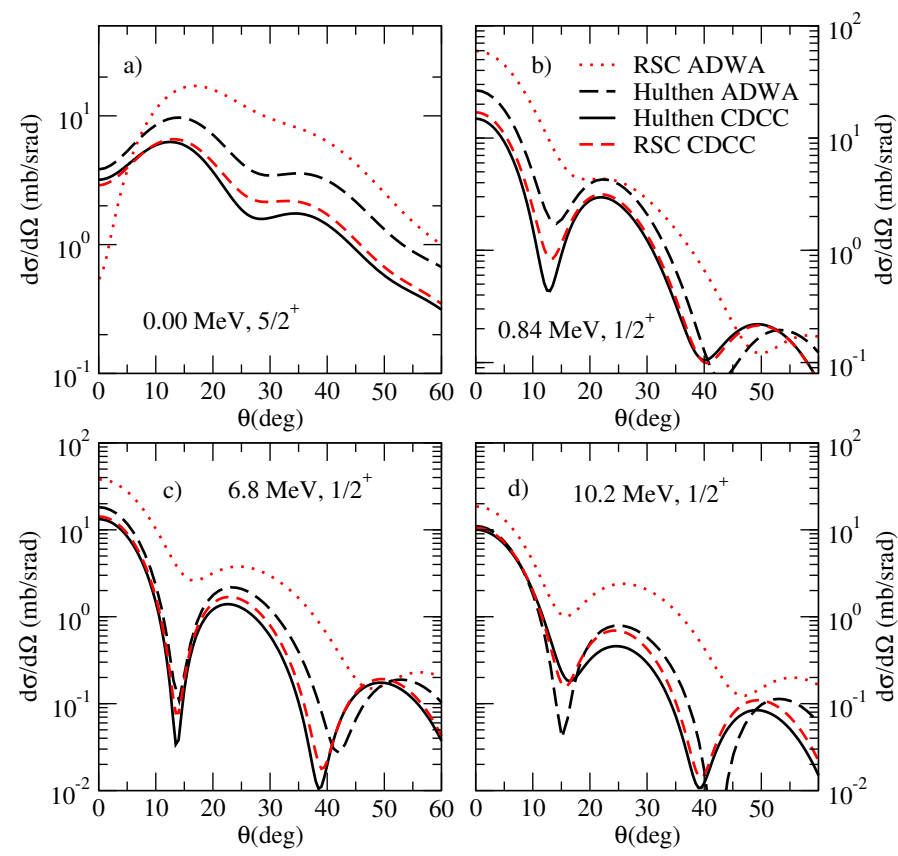

FIG. 2. The same as in Fig. 1 but for $E_{d}^{\text {lab }}=50 \mathrm{MeV}$. matrix element $\left\langle T_{n p}\right\rangle_{V}$ associated with these potentials [1]. The Hulthén-ADWA calculations effectively include only $s$-wave continuum while RSC-ADWA includes the $d$-wave continuum as well. To check to what extent the difference between these calculations is due to the missing $s$-wave continuum we performed the Hulthén-CDCC calculations with $s$-wave bins only for one selected case, $J^{\pi}=5 / 2^{+}$at $E_{d}^{\mathrm{lab}}=9.2 \mathrm{MeV}$. The cross sections were $10 \%$ lower thus pointing that the $n-p$ model dependence in ADWA partially originates from a different $d$-wave content of continuum associated with these models.

The CDCC calculations show that the sensitivity to the $n$ - $p$ model is significantly reduced. It is less than $4 \%$ for $E_{d}^{\mathrm{lab}}=9.2 \mathrm{MeV}$ but can understandably increase with the deuteron incident energy up to $14 \%$.

Although our main aim is the comparison of ADWA and CDCC calculations, given the existence of experimental data for ${ }^{27} \mathrm{Al}\left(1 / 2^{+}\right)$[20], we deduced spectroscopic factors from these data using both ADWA and CDCC and both NN potentials. They are presented in Table II and compared to previous ADWA calculations with local optical potentials. Both CDCC calculations and the Hulthén-ADWA reproduce the shape of experimental data but RSC-ADWA overestimates the data at larger angles for the states at $E_{x}=0.84$ and 6.8 $\mathrm{MeV}$. The spectroscopic factors extracted with CDCC are larger than those determined in [20], but this difference decreases with the excitation energy.

Understanding reduced sensitivity. The strong sensitivity of the ADWA cross sections to the NN model comes from the coefficient $M_{0}$ in the transcendental equation for the local-equivalent adiabatic potential $U^{\text {loc }}$,

$$
U^{\text {loc }}=M_{0}\left(U_{n A}+U_{p A}\right) \exp \left[-\gamma\left(E-U_{C}-U^{\text {loc }}\right)\right],
$$

TABLE II. Spectroscopic factors obtained from the ${ }^{26 m} \mathrm{Al}(d, p)^{27} \mathrm{Al}\left(1 / 2^{+}\right)$cross sections calculated with ADWA and CDCC with two different NN potentials, RSC and Hulthén for $E_{d}^{\text {lab }}=9.2 \mathrm{MeV}$. All energies are in $\mathrm{MeV}$.

\begin{tabular}{llllll}
$E_{x}$ & $\begin{array}{l}\text { ADWA } \\
\text { Hulthén }\end{array}$ & $\begin{array}{l}\text { ADWA } \\
\text { RSC }\end{array}$ & $\begin{array}{l}\text { CDCC } \\
\text { Hulthén }\end{array}$ & $\begin{array}{l}\text { CDCC } \\
\text { RSC }\end{array}$ & $\begin{array}{l}\text { Ref } \\
{[20]}\end{array}$ \\
\hline \hline 0.84 & 0.07 & - & 0.13 & 0.11 & 0.08 \\
6.8 & 0.14 & - & 0.14 & 0.14 & 0.11 \\
10.2 & 0.13 & 0.08 & 0.18 & 0.18 & 0.16 \\
\hline \hline
\end{tabular}


where $\gamma$ is a constant [11]. This coefficient is given by

$$
M_{0}=N \int d \boldsymbol{s} d \boldsymbol{x} H(s) \phi_{d}^{*}\left(\boldsymbol{x}+\alpha_{1} s\right) V_{n p}(\boldsymbol{x}) \phi_{d}(\boldsymbol{x})
$$

with $N=\left\langle\phi_{d}\left|V_{n p}\right| \phi_{d}\right\rangle^{-1}$ (see [11] for the link between $M_{0}$ and $\left\langle T_{n p}\right\rangle_{V}$ ). Because of the short range of $V_{n p} \phi_{d}$, $M_{0}$ is highly sensitive to the details of $\phi_{d}$ at small $x$. In the CDCC, the main channel corresponds to the folding model with the $U^{\text {loc }}$ found from Eq. (21) and $M_{0}$ generated by Eq. (22) with $N=1$ and without $V_{n p}$ [24]:

$$
M_{0}=\int d \boldsymbol{x} \bar{\phi}_{d}^{*}(\boldsymbol{x}) \phi_{d}(\boldsymbol{x}) \text {. }
$$

Because of the small deuteron binding energy this $M_{0}$ is determined by the large values of $x$, corresponding to small $n$ - $p$ momenta, where all the NN models agree. Also, because of the small range of nonlocality $\beta$, in this range $\bar{\phi}_{d} \approx \phi_{d}$ (see Fig. 3a) and, therefore, $M_{0} \approx 1$. The same statements are relevant for low-energy continuum bins which are affected by the nonlocality and differences in the NN potentials only at small $x$ (Fig. 3b) thus explaining the reduced sensitivity to the deuteron model in the $(d, p)$ calculations with CDCC. The differences in the NN model affect high-energy bins ( Fig. 3c) where modifications due to nonlocality are stronger. As a result, the sensitivity to the high $n-p$ momenta is stronger for a large deuteron incident energy, as seen from Table I.

The ADWA could be corrected by including more Weiberg states in the expansion of $\Phi(\boldsymbol{R}, \boldsymbol{r})$ [8, 25]. This would involve calculations of nondiagonal localequivalent coupling potentials $U_{i i^{\prime}}^{\text {loc }}$ that depend on the coefficients given by (22) but with Weinberg states $\phi_{i}^{W}$ instead of $\phi_{d}$. Such coefficients (and, therefore, the $U_{i i^{\prime}}^{\text {loc }}$ and the corresponding $(d, p)$ cross sections) would be determined by the model-dependent short-range behaviour of $V_{n p} \phi_{i}^{W}$. It was shown in [26] that continuum bins could be expanded over Weinberg states. Therefore, sufficient number of NN-dependent Weinberg states should recover the almost-NN-independent CDCC calculations. It is worth mentioning that for local optical potentials the non-adiabatic corrections explicitly depend on the same NN model-dependent matrix element $\left\langle T_{n p}\right\rangle_{V}[27]$ that features in the nonlocal ADWA.

Conclusions. Based on our newly developed localequivalent CDCC model with nonlocal optical potentials, we have shown that the previously reported strong sensitivity of the adiabatic $(d, p)$ cross sections, calculated with nonlocal nucleon optical potentials, is significantly reduced. For low deuteron incident energies it is now less than $4 \%$ but can increase up to $14 \%$ for higher energies.

We have also found that non-adiabatic effects are much stronger than those in the case of local optical potentials. To confirm this finding, the nonlocal CDCC should be extended beyond the leading order. Exact ADWA cross sections with nonlocal potentials are smaller than the leading-order cross sections [12] but this tendency may not necessarily be the same in the CDCC case. It is conceivable that the difference between exact nonlocal
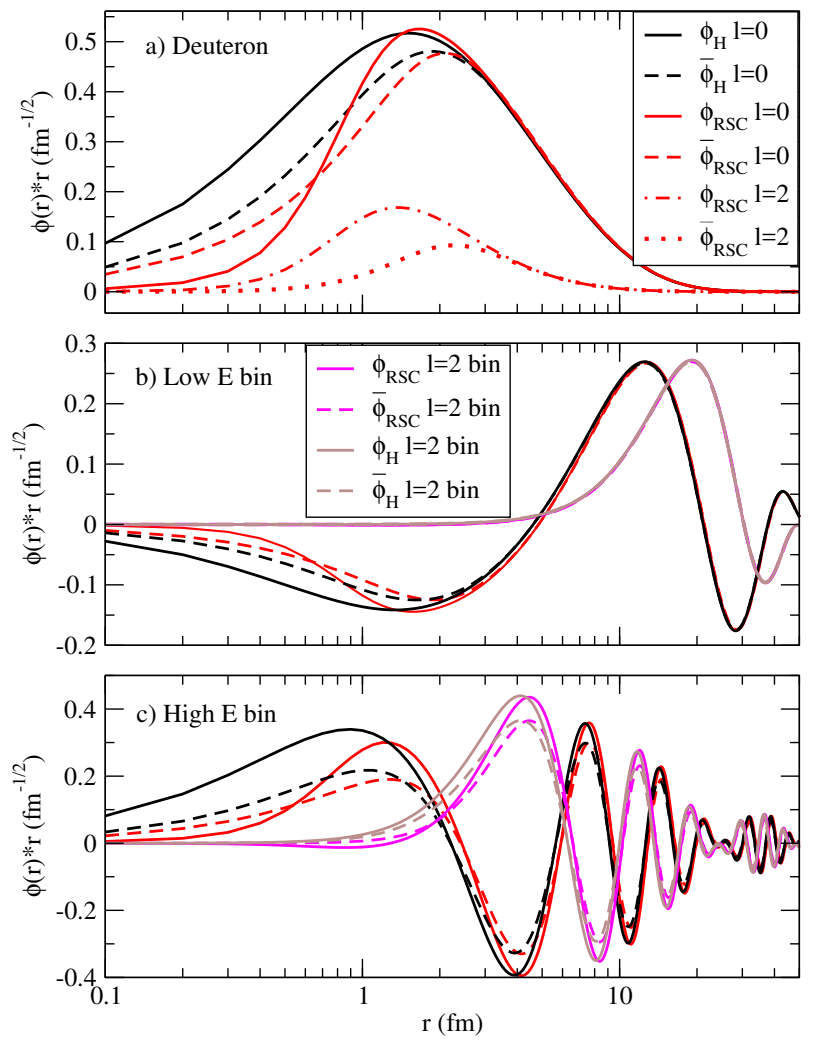

FIG. 3. Original $\phi_{i}$ and modified-by-nonlocality $\bar{\phi}_{i}$ deuteron (a) and $J^{\pi}=1^{+}$bin $(b, c)$ wave functions obtained with RSC and Hulthén potentials. The low-energy bin (b) has an everage energy $\bar{E}=1.6 \mathrm{MeV}$ with a spread of $\Delta E=2 \mathrm{MeV}$ while the high-energy bin (c) corresponds to $\bar{E}=34.375 \mathrm{MeV}$ and $\Delta E=19.25 \mathrm{MeV}$. For both energies, the bins with incoming $s$ and $d$ waves are presented.

CDCC and nonlocal ADWA can be smaller than that obtained in this work.

The sensitivity to high $n-p$ momenta due to uncertainties of the adiabatic approximation suggests that theoretical analysis of $(d, p)$ experiments should be extended beyond the adiabatic approximation when nonlocal optical potentials are used. This is an important message given the current interest of other groups in ADWA with nonlocal potentials, such as in $[28,29]$. Full nonlocal CDCC calculations could help to refine the spectroscopic factors and asymptotic normalization coefficients obtained from $(d, p)$ reactions. We note that present results were obtained with energy-independent optical potentials. A proper treatment of energy-dependence within the threebody context is a challenge, in particular in the CDCC formalism, where the energy between nucleon and target is not well defined in the considered final states. Whether approximate prescriptions to take this dependence into account could result in additional $\mathrm{NN}$-model dependence of $(\mathrm{d}, \mathrm{p})$ cross sections remains to be investigated.

Acknowledgements. We are grateful to R. C. Johnson and A. M. Moro for fruitful discussions. This work was supported by the United Kingdom Science and 
Technology Facilities Council (STFC) under Grant No. ST/L005743/1. M. G.-R. acknowledges a research grant from the Spanish Ministerio de Educación, Cultura y Deporte, Ref: FPU13/04109.
[1] G.W. Bailey, N.K. Timofeyuk and J.A. Tostevin, Phys. Rev. Lett. 117, 162502 (2016); PRL 119, 159901(E) (2017).

[2] V. Margerin et al, Phys. Rev. Lett. 115, 062701 (2015)

[3] L. Hulthén and M. Sugawara, Handbuch der Physik. Springer, 1957

[4] R.B. Wiringa, V.G.J. Stoks and R. Schiavilla, Phys. Rev. C. 51, 38 (1995).

[5] V. G. J. Stoks et al, Phys. Rev. C 49, 2950 (1994)

[6] R. Machleidt, Phys. Rev. C. 63024001 (2001).

[7] E. Epelbaum, H. Krebs and U.-G. Meißner, Phys. Rev. Lett. 115122301 (2015).

[8] R. C. Johnson and P.C. Tandy, Nucl. Phys. A235, 56 (1974)

[9] R.C. Johnson and P.J.R. Soper, Phys. Rev. C 1, 976 (1970)

[10] N. K. Timofeyuk and R. C. Johnson, Phys. Rev. Lett. 110, 112501 (2013).

[11] N.K. Timofeyuk and R.C. Johnson, Phys. Rev. C87, 064610 (2013)

[12] G.W. Bailey, N.K. Timofeyuk and J.A. Tostevin, Phys. Rev. C95, 024603 (2017).

[13] G. H. Rawitscher, Phys. Rev. C9, 2210 (1974).

[14] N. Austern, Y. Iseri, M. Kamimura, M. Kawai, G. Rawitscher, and M. Yahiro, Phys. Rep. 154, 125 (1987).

[15] N. J. Upadhyay, A. Deltuva, and F. M. Nunes,
Phys.Rev.C85, 054621 (2012).

[16] D. Y. Pang and A. M. Mukhamedzhanov, Phys.Rev.C90, 044611 (2014).

[17] Y. Chazono et al, Phys. Rev. C95, 064608 (2017)

[18] F. Perey and B. Buck, Nucl. Phys. 32, 353 (1962).

[19] I.J. Thompson, Comput. Phys. Rep. 7, 167 (1988)

[20] S. Almaraz-Calderon et al, Phys. Rev. Lett. 119, 072701 (2017)

[21] M. M. Giannini and G. Ricco, Ann. Phys. (NY) 102, 458 (1976).

[22] O.A. Rubtsova, private communication of her unpublished CDCC calculations with closed channels for one selected $(d, p)$ reaction at $E_{d}=9 \mathrm{MeV}$ performed using the technique from [23]

[23] V. I. Kukulin and O. A. Rubtsova, Phys. Rev. C 76, 047601 (2007)

[24] R.C. Johnson and P.J.R. Soper, Nucl. Phys. A182, 619 (1972)

[25] A.Laid, J.A.Tostevin, R.C.Johnson, Phys. Rev. C48, 1307 (1993)

[26] D.Y.Pang, N.K.Timofeyuk, R.C.Johnson, J.A.Tostevin, Phys.Rev. C 87, 064613 (2013)

[27] R.C. Johnson, J. Phys. G 41, 094005(2014)

[28] L.Titus and Nunes, Phys. Rev. C93, 014604 (2016)

[29] A.Ross, L.J.Titus, F.M.Nunes, Phys.Rev. C 94, 014607 (2016) 